\title{
Follow-up of atheroma burden with sequential whole body contrast enhanced MR angiography: a feasibility study
}

\author{
Jonathan R. Weir-McCall ${ }^{1}\left(D_{0} \cdot\right.$ Richard D. White $^{2} \cdot$ Prasad G. Ramkumar $^{3}$. \\ Stephen J. Gandy ${ }^{4}$. Faisel Khan ${ }^{1} \cdot$ Jill J. F. Belch ${ }^{1} \cdot$ Allan D. Struthers $^{1}$ • \\ J. Graeme Houston ${ }^{1,3}$
}

Received: 25 November 2015/ Accepted: 13 January 2016/Published online: 25 January 2016

(c) The Author(s) 2016. This article is published with open access at Springerlink.com

\begin{abstract}
Assess the feasibility of whole body magnetic resonance angiography (WB-MRA) for monitoring global atheroma burden in a population with peripheral arterial disease (PAD). 50 consecutive patients with symptomatic PAD referred for clinically indicated MRA were recruited. Whole body MRA (WB-MRA) was performed at baseline, 6 months and 3 years. The vasculature was split into 31 anatomical arterial segments. Each segment was scored according to degree of luminal narrowing: $0=$ normal, $1=<50 \%, \quad 2=50-70 \%, \quad 3=71-99 \%, \quad 4=$ vessel occlusion. The score from all assessable segments was summed, and then normalised to the number of assessable vessels. This normalised score was divided by four (the maximum vessel score) and multiplied by 100 to give a final standardised atheroma score (SAS) with a score of 0-100. Progression was assessed with repeat measure ANOVA. 36 patients were scanned at 0 and 6 months, with
\end{abstract}

Jonathan R. Weir-McCall

jweirmccall@gmail.com

Richard D. White

dr.r.white@gmail.com

Prasad G. Ramkumar

prasad.guntur@nhs.net

Stephen J. Gandy

Stephen.gandy@nhs.net

Faisel Khan

f.khan@dundee.ac.uk

Jill J. F. Belch

j.j.f.belch@dundee.ac.uk

Allan D. Struthers

a.d.struthers@dundee.ac.uk

J. Graeme Houston

Ghouston@nhs.net
26 patients scanned at the 3 years follow up. Only those who completed all three visits were included in the final analysis. Baseline atherosclerotic burden was high with a mean SAS of $15.7 \pm 10.3$. No significant progression was present at 6 months (mean SAS $16.4 \pm 10.5, p=0.67$ ), however there was significant disease progression at 3 years (mean SAS $17.7 \pm 11.5, p=0.01$ ). Those with atheroma progression at follow-up were less likely to be on statin therapy (79 vs $100 \%, p=0.04$ ), and had significantly higher baseline SAS $(17.6 \pm 11.2$ vs $10.7 \pm 5.1$, $p=0.043$ ). Follow up of atheroma burden is possible with WB-MRA, which can successfully quantify and monitor atherosclerosis progression at 3 years follow-up.

Keywords Atherosclerosis - Peripheral arterial disease . Whole-body imaging - Magnetic resonance angiography . Disease progression

1 Division of Cardiovascular and Diabetes Medicine, Medical Research Institute, University of Dundee, Dundee DD1 9SY, UK

2 Department of Clinical Radiology, University Hospital of Wales, Cardiff CF14 4XW, UK

3 NHS Tayside Clinical Radiology, Ninewells Hospital, Dundee DD1 9SY, UK

4 NHS Tayside Medical Physics, Ninewells Hospital, Dundee DD1 9SY, UK 


\section{Introduction}

Atherosclerosis is the pathological process underpinning the leading causes of morbidity and mortality in the western world. Despite the fact that it typically presents with symptoms localising to a single site, atherosclerosis is a systemic process, with those with disease presenting in one site at markedly increased risk for developing symptomatic disease at another site [1]. Peripheral arterial disease (PAD) is particularly prone to this with previous reports showing a high prevalence of silent myocardial infarctions, extra-site disease, and risk of future cardiovascular events [2-4].

Whole body magnetic resonance angiography (WBMRA) has been proven to be useful for assessing the atheroma burden throughout the body in both healthy volunteers and patients with cardiovascular disease [5-7]. This has been shown to correlate well with the presence of obstructive coronary arterial disease, and as a predictor of future major adverse cardiovascular events [8-10]. However the ability to assess and quantify atheroma progression with WB-MRA has not been previously investigated. Therefore we set out to determine the feasibility of using WB-MRA to quantify and monitor atheroma progression in a population with $\mathrm{PAD}$.

\section{Materials and methods}

Institutional review board and research and development committee approval was granted for this study. All enrolled participants gave written informed consent. 50 consecutive patients referred for MRA of their lower limbs with PAD and symptomatic claudication were recruited. Disease severity was classified using the Fontaine scoring system. All patients underwent WB-MRA at baseline, with repeat WB-MRA scans being performed at 6 months, and 3 years after baseline. The baseline data of the full cohort have been described in detail previously, while the current article focuses on the longitudinal follow-up and assessment of the cohort [11].

\section{WB-MRA}

At baseline and 6 months, all scanned patients underwent contrast-enhanced WB-MRA using a 1.5T MRI unit (Siemens Magnetom Avanto, Erlangen, Germany). Fifty consecutive patients were recruited to the study. Four patients were excluded due to inability to complete the MRI examination due to claustrophobia, leaving 46 participants who received the baseline scan. A total of 36 participants completed the 6 months follow-up study, and 26 returned for the 3 years follow-up scan. Drop outs were due to: two deaths; eight who had developed subsequent health complications and felt unable to continue; and ten who were uncontactable. At 3 years follow-up, 26 participants were scanned: $\mathrm{n}=8$ were scanned at $1.5 \mathrm{~T}$ and $\mathrm{n}=18$ were scanned on a 3T MRI unit (Siemens Magnetom Trio, Erlangen, Germany).

Patients were imaged head first and supine in the magnet bore, with surface coils used to cover the entire body as follows: one head matrix; one neck matrix; one spine matrix; two body matrix; and one peripheral angiography. WB-MRA was performed using four separate stations for image acquisition, as below:

Station 1-head, neck and thoracic vessels

Station 2-abdominopelvic vessels

Station 3-upper leg vessels

Station 4-lower leg vessels

Full details of the technique are described in detail elsewhere [11], but in brief, the examination consisted of precontrast ('mask') and post-contrast MR imaging at all anatomical stations using a fast spoiled gradient echo (FLASH-Siemens, Germany) sequence. After the precontrast images for station 1 and 4 had been acquired, $20 \mathrm{ml}$ of $0.5 \mathrm{M}$ intravenous gadolinium based contrast agent (gadoterate meglumine, Guerbet, FR) followed by a $20 \mathrm{ml}$ volume of normal saline was administered at $1 \mathrm{ml} / \mathrm{s}$ using an injector pump via a $20 \mathrm{G}$ venflon in the antecubital fossa using a dual injection method [12]. Following this, a fluoroscopic-guided post-contrast FLASH sequence was implemented at station 1 when the contrast agent reached the aortic arch. Immediately thereafter, the same FLASH acquisition was completed at station 4 on three consecutive occasions (to optimise the peak contrast enhancement at the lower limb vessels).

After a 10 min delay, the pre-contrast mask for stations 2 and 3 were acquired following which the second injection of contrast agent was delivered and flouroscopic guided triggering was used at the proximal abdominal aorta to define the start of the FLASH acquisition for station 2 . Finally, FLASH data for station 3 were acquired immediately after completion of the station 2 sequence.

The resulting images were analysed by dividing the arterial tree into 31 distinct anatomical segments. Each segment was scored according to the maximal stenosis present at any point within it, using a 5 point scoring system:

- 0: Normal vessel

- $1:<50 \%$ stenosis

- 2: 50-70\% stenosis

- 3: $>70 \%$ stenosis

- 4: Completely occluded vessel 
Arterial segments which were not visualised with sufficient clarity for grading of the degree of stenosis were not analysed. To account for this, the final score was divided by the number of segments which had been successfully analysed (n), and then calculated as a percentage of the maximum possible score (see equation below) to produce a 'standardised atheroma score' (SAS) [13].

$S A S=\left[\left(\frac{\sum \text { vessel scores }}{n}\right) \times \frac{1}{4}\right] \times 100$

The 31 vessel segments were also subdivided into 5 anatomical territories: (1) the head and neck arteries; (2) the aorta; (3) the abdominal arteries; (4) the ilio-femoral arteries; and (5) popliteal and infrageniculate arteries (Fig. 1). Regional SASs were calculated for each anatomical territory.

WB-MRA analysis was performed using a diagnostic PACS radiological workstation (Carestream PACS Client Suite Version 10.1 sp1, Rochester, NY, USA) using the raw data in multiplanar reformat (MPR) analysis. Multiplanar visualisation was used in order to best visually estimate the degree of area stenosis. All images were analysed by a single radiologist with over 3 years experience in vascular radiology blinded to the patients' clinical details. In order to determine repeatability of the scoring 26 scans from the final study visit were scored twice by this observer with a greater than 6 months interval between readings, and by two additional observers (one with over 3 years, and the other with over 20 years experience in vascular radiology). 2-way mixed, absolute agreement, average measure intraclass correlation co-efficient (ICC) for the whole body SAS was 0.94 (95 \% CI 0.82-0.98) for intra-observer repeatability, and 0.89 (95 \% CI 0.63-0.96) for inter-observer variability between the 3 observers (ICC $>0.75=$ excellent, $0.4-0.75=$ good, $<0.4=$ poor, $<0.2=$ slight $)$.

\section{Ankle Brachial Pressure Index (ABPI) and carotid intima media thickness (CIMT)}

These were performed as previously described [11]. Briefly, the CIMT was measured $1 \mathrm{~cm}$ proximal to the carotid bulb at a point where $1 \mathrm{~cm}$ of continuous intima media could be visualised. Both common carotids were measured twice and an average obtained.

ABPI was calculated using the highest of the two upper limb brachial blood pressures, and the higher of the two lower limb blood pressures.

\section{Statistical analysis}

Categorical variables are presented as frequencies or percentages; normally distributed continuous variables are presented as means $\pm \mathrm{SD}$; and skewed variables are presented as median with interquartile range (IQR; $25-75$ th percentile). Normality of continuous variables was tested with the Shapiro-Wilk test. An independent sample $t$ test was used to compare demographics between those with and without atheroma progression. Repeated measure ANOVA

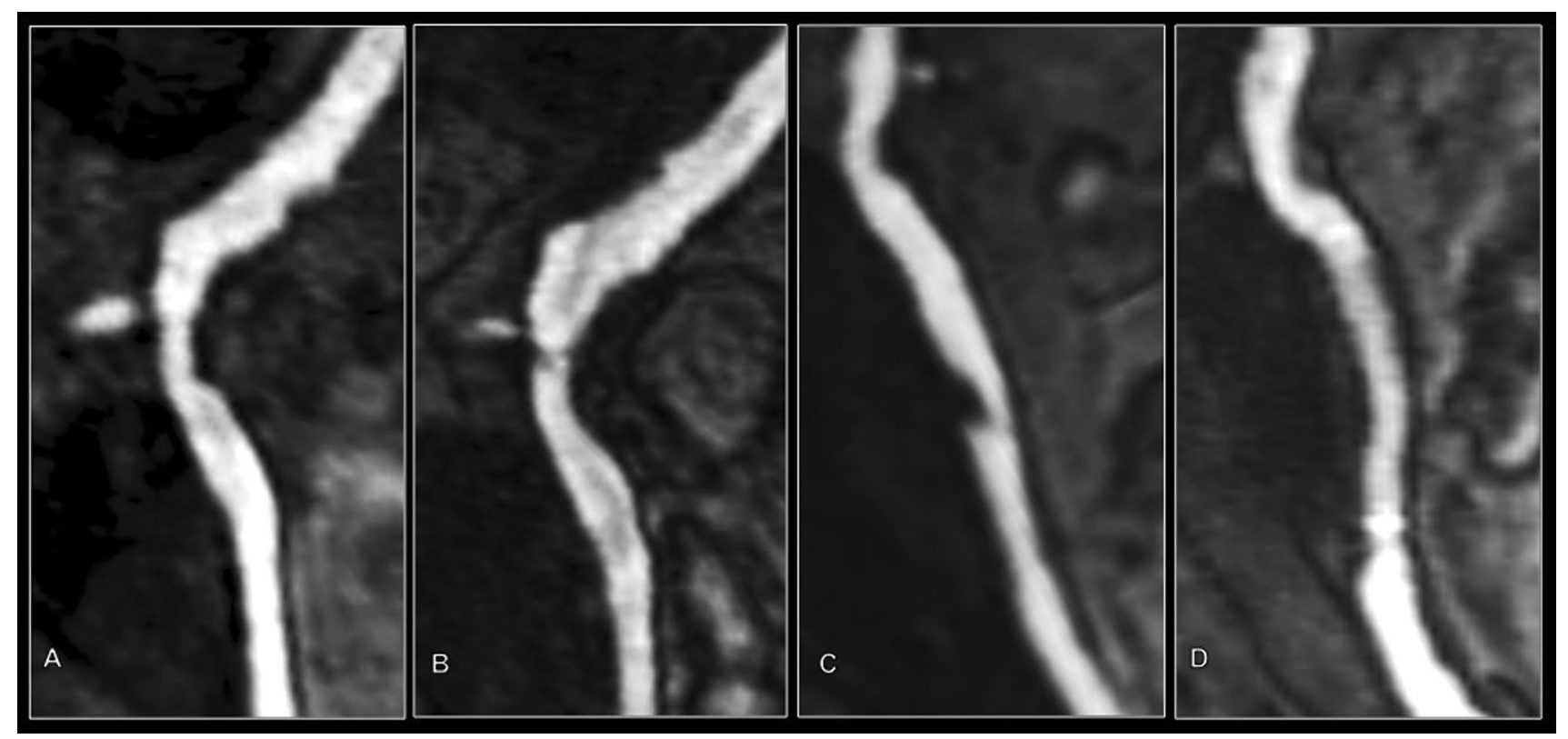

Fig. 1 Examples of atheroma progression $(\mathbf{a}, \mathbf{b})$ and regression $(\mathbf{c}, \mathbf{d})$. MRA of the right iliac artery at baseline (a) showing a grade 2 $(50-70 \%)$ stenosis just distal to the internal iliac artery origin which has progressed to a grade $3(70-99 \%)$ stenosis at 3 years follow-up (b). MRA of the iliac artery in patient with interval angioplasty showing baseline grade 3 stenosis (c) which resolves on follow-up with only minor remaining luminal irregularity (grade 1) (d) 
was used to analyse SAS progression between the baseline and 3 years follow up visit, with a post hoc analysis with bonferonni correction for differences between the three visits. A $p$ value of $<0.05$ was considered significant. All statistical analyses were performed with IBM SPSS Statistics for Windows, version 21.0 (IBM Corp., NY, USA).

\section{Results}

Fifty consecutive patients were recruited to the study. Four patients were excluded due to inability to complete the MRI examination due to claustrophobia, leaving 46 participants who received the baseline scan. A total of 36 participants completed the 6 months follow-up study, and 26 returned for the 3 years follow-up scan. Drop outs were due to: two deaths; eight who had developed subsequent health complications and felt unable to continue; ten who were uncontactable. Of the final 26, $73 \%(\mathrm{n}=19)$ were male, with a mean age of $64.5 \pm 9.5$ years. A summary of demographic data is provided in Table 1. In terms of symptomatic PAD severity, 2 patients were Fontaine 1, 2 were Fontaine IIa, 35 were Fontaine IIb, 5 were Fontaine III and 2 were Fontaine IV. There was no significant differences in the demographics or baseline atheroma burden between those who completed all three visits and those who dropped out ( $p>0.1$ for all variables).

There were 2418 segments for analysis. Of the 2418 possible segments, 2387 (98.7\%) were considered to be of sufficient quality for analysis. At baseline SAS was $15.7 \pm 10.3$, with a mean SAS of $16.4 \pm 10.5$ at 6 months, and a mean SAS of $17.7 \pm 11.5$ at 3 years. No significant progression was present at 6 months with a change in SAS of $0.63 \pm 0.51(p=0.67)$. This lack of significant progression persisted even when all 36 who underwent the first two scans were included in the analysis (mean progression $0.96 \pm 1.08, p=0.38)$. There was significant disease progression at 3 years with a mean progression in SAS of $1.92 \pm 1.24(p=0.01)$. Over the 3 years period, this gave an annual progression rate in the atheroma burden of $4.1 \%$ per year. Significant progression was also present between the 6 months SAS and the 3 years follow-up (mean progression $1.29 \pm 0.84, p=0.016$ ) - see Fig. 2 .

At 3 years eighteen participants showed progression in their atheroma score, while two were stable, and six showed an improvement (see Fig. 3). Out of the six whose atheroma score reduced, three had undergone one or more percutaneous interventions (one with femoral angioplasty, one with bilateral iliac angioplasties, one with bilateral femoral angioplasties with unilateral stent insertion). One patient had a single intervention between visit 1 and 2, one had a single intervention between visit 2 and 3 , and one had
Table 1 Demographics and clinical characteristics of the study population

\begin{tabular}{ll}
\hline & Study population $(\mathrm{n}=26)$ \\
\hline Male $(\%)$ & $19(73 \%)$ \\
Age $($ years $)$ & $64.5 \pm 9.6$ \\
BMI $\left(\mathrm{kg} / \mathrm{m}^{2}\right)$ & $28.4(20.7-41.2)$ \\
Systolic BP $(\mathrm{mmHg})$ & $145 \pm 11$ \\
Diastolic BP $(\mathrm{mmHg})$ & $82(43-102)$ \\
Type 2 diabetes & $5(19 \%)$ \\
Hypertension & $18(69 \%)$ \\
Smoking status & $5(19 \%)$ \\
Current smoker & $19(73 \%)$ \\
Ex-smoker & $2(8 \%)$ \\
Non-smoker & $38 \pm 34$ \\
Smoking pack years & $19(73 \%)$ \\
Medications & $22(85 \%)$ \\
Anti-hypertensive & $22(85 \%)$ \\
Anti-platelet & $15.7 \pm 10.3$ \\
Statin & $0.94 \pm 0.18$ \\
Baseline SAS & $0.82(0.51-2.05)$ \\
CIMT &
\end{tabular}

Values expressed as mean $\pm \mathrm{SD}$, median (range) or $\mathrm{N}(\%)$

$B M I$ body mass index, $B P$ blood pressure, $S A S$ standardised atheroma score, CIMT carotid intima media thickness, $A B P I$ ankle-brachial pressure index

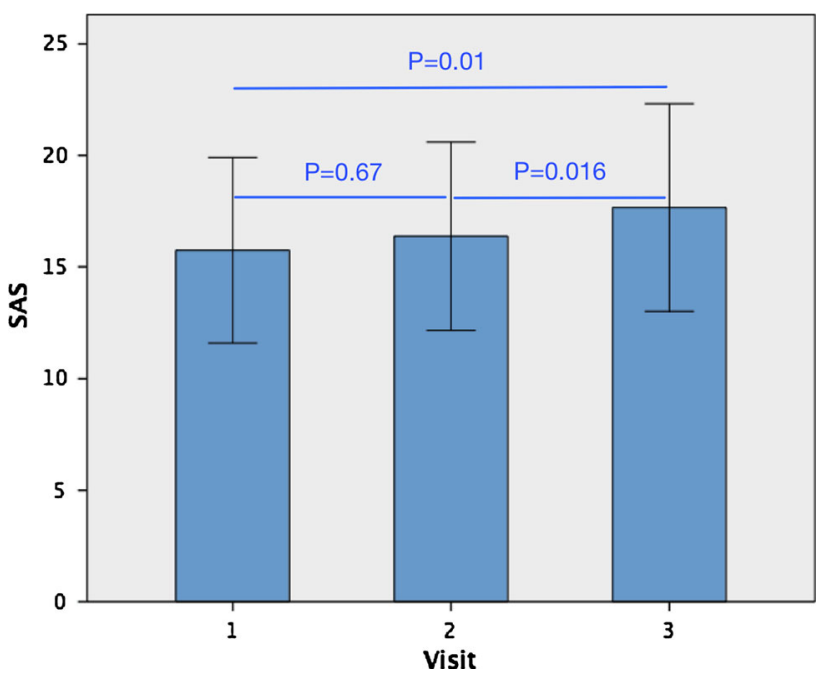

Fig. 2 Comparison of atheroma score at baseline, 6 months and 3 years. SAS standardized atheroma score. T-bars represent $95 \%$ confidence intervals

2 separate visits for interventional procedures with one between each visit. The remaining three had no intervention to explain their improvement. In comparison two participants demonstrated atheroma progression despite interval percutaneous intervention (one with two 


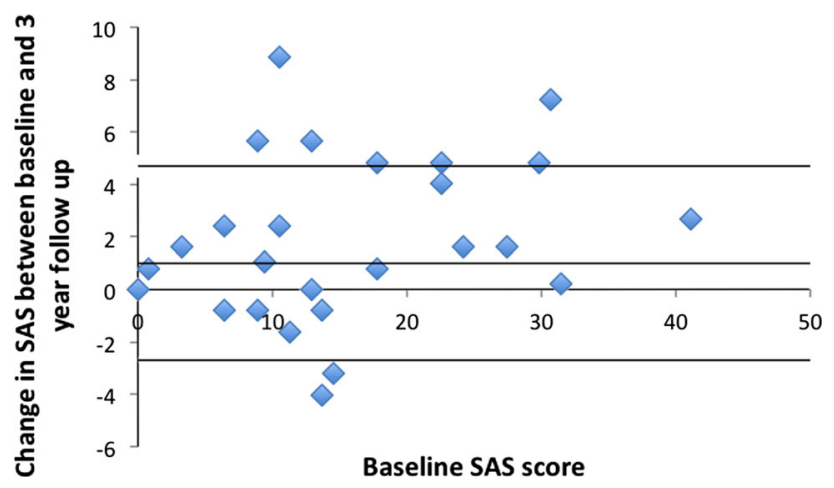

Fig. 3 Bland Altman plot comparing baseline SAS (x-axis) with SAS change at 3 years $(y$-axis). The mid, upper and lower line represents the mean, and upper and lower $1.96 * \mathrm{SD}$, of the change in SAS between baseline and 3 years follow up respectively

angioplasties and a stent inserted between the first two scans, the other with a common iliac stent inserted between the first and second scan). When the participants who were scanned at $1.5 \mathrm{~T}$ on the final visit were compared with those scanned at $3 \mathrm{~T}$ on the third visit there was no difference in the final visit atheroma burden $(1.5 \mathrm{~T}: 16.6 \pm 9.2,3 \mathrm{~T}$ $18.1 \pm 12.6, p=0.75$ ) or rate of progression (1.5T: $1.7 \pm 2.7,3 \mathrm{~T} 2.2 \pm 3.2, p=0.71$ ).

Whilst the whole body SAS demonstrated significant progression over the 3 years, when this was split into five distinct anatomical regions, no single region demonstrated significant progression on its own over the 3 years [(1) head and neck (mean difference 1.4 $\pm 4.7, p=0.4$ ); (2) aorta (mean difference $1.9 \pm 4.3, p=0.09$ ); (3) the abdominal arteries (mean difference $0.96 \pm 8.9, p=01$ ); (4) ilio-femoral arteries (mean difference $2.5 \pm 5.1$, $p=0.16$ ); and (5) popliteal and infrageniculate arteries (mean difference $3.0 \pm 3.0, p=0.15$ ), (see Table 2)].

Out of the baseline characteristics, statin therapy was significantly more common in the group showing no progression than those with progression (100 vs $79 \%$, $p=0.04)$. In addition, baseline SAS was significantly higher in those who progressed compared with those with stable disease/atheroma regression $(17.6 \pm 11.2$ vs $10.7 \pm 5.1, p=0.043$ ) (see Table 3).
Table 3 Comparison of baseline characteristics between those with and without atheroma progression at 3 years follow-up

\begin{tabular}{llll}
\hline & Progression & Stable/regression & $p$ \\
\hline $\mathrm{N}$ & $18(69 \%)$ & $8(31 \%)$ & \\
Male $(\%)$ & $15(79 \%)$ & $4(57 \%)$ & 0.29 \\
Age (years) & $66.3 \pm 10.3$ & $59.6 \pm 4.7$ & 0.11 \\
BMI $\left(\mathrm{kg} / \mathrm{m}^{2}\right)$ & $28.7(20.7-36.5)$ & $28(24.9-41.2)$ & 0.41 \\
Systolic BP $(\mathrm{mmHg})$ & $144.8 \pm 11.4$ & $148.6 \pm 11.9$ & 0.47 \\
Diastolic BP $(\mathrm{mmHg})$ & $81(43-102)$ & $84(68-95)$ & 0.57 \\
Pulse pressure & $64.5 \pm 14.3$ & $65.9 \pm 15.5$ & 0.83 \\
Hypertension & $13(68 \%)$ & $5(71 \%)$ & 0.89 \\
Type 2 diabetes & $3(16 \%)$ & $2(29 \%)$ & 0.48 \\
Current smoker & $4(21 \%)$ & $1(14 \%)$ & 0.71 \\
Ex-smoker & $14(74 \%)$ & $5(71 \%)$ & 0.91 \\
Non-smoker & $1(5 \%)$ & $1(14 \%)$ & 0.46 \\
Smoking pack years & $30.4 \pm 21.6$ & $55.4 \pm 50.1$ & 0.1 \\
Anti-hypertensive & $13(68 \%)$ & $6(86 \%)$ & 0.35 \\
Anti-platelet & $17(89 \%)$ & $5(71 \%)$ & 0.39 \\
Statin & $15(79 \%)$ & $7(100 \%)$ & $\mathbf{0 . 0 4}$ \\
Baseline SAS & $17.6 \pm 11.2$ & $10.7 \pm 5.1$ & $\mathbf{0 . 0 4 3}$ \\
CIMT & $0.94 \pm 0.19$ & $0.93 \pm 0.18$ & 0.92 \\
ABPI & $0.8(0.51-2.05)$ & $0.84(0.72-1.37)$ & 0.85 \\
\hline
\end{tabular}

The bold values indicate statistically significant $(p<0.05)$

Values expressed as mean $\pm \mathrm{SD}$, median (range) or $\mathrm{N}(\%)$

$B M I$ body mass index, $B P$ blood pressure, $S A S$ standardised atheroma score, CIMT carotid intima media thickness, $A B P I$ ankle-brachial pressure index

\section{Discussion}

In this feasibility study we have demonstrated the possibility of using WB-MRA to monitor and quantify atherosclerosis progression in a population with PAD. To the best of the authors knowledge, this is the first time that sequential WB-MRA has been used to quantify the progression rates of stenotic arterial disease. This adds to the current literature which shows that WB-MRA can detect significant arterial pathology outwith the lower limbs, more accurately quantifies stenotic disease in the lower limbs
Table 2 Change in whole body and regional SAS between baseline and 3 years follow-up

\begin{tabular}{lclcl}
\hline & Baseline & 3 years & Mean difference & $p$ \\
\hline WB-SAS & $15.7 \pm 10.3$ & $17.7 \pm 11.5$ & $2.0 \pm 3.1$ & 0.014 \\
Head/neck-SAS & $9.0 \pm 11.2$ & $10.4 \pm 11.7$ & $1.4 \pm 4.7$ & 0.4 \\
Aorta-SAS & $13.5 \pm 8.5$ & $15.4 \pm 6.9$ & $1.9 \pm 4.3$ & 0.09 \\
Abdomen-SAS & $9.2 \pm 12.5$ & $10.2 \pm 11.7$ & $0.96 \pm 8.9$ & 1 \\
Ilio-femoral-SAS & $32.2 \pm 17.5$ & $34.3 \pm 19.7$ & $2.5 \pm 5.1$ & 0.16 \\
Run off-SAS & $15.8 \pm 19.6$ & $18.8 \pm 22.2$ & $3.0 \pm 3.0$ & 0.15 \\
\hline
\end{tabular}

Values expressed as mean $(95 \% \mathrm{CI})$

SAS standardised atheroma score 
than ABPI, and can effect management changes in this population [3, 14, 15]. Additionally, the extra cost of extending standard lower limb MRA to include whole body imaging in combination with a cardiac MRI has been shown to be cost-effective due to a reduction in the requirement for down stream extra tests [16].

The ability to quantify and monitor atherosclerosis is desirable for several reasons. Previous studies have shown atheroma burden to correlate better with future cardiovascular events than traditional risk factors, and therefore holds potential as a useful prognostic marker. Furthermore, the ability to identify early changes in a small number of people holds potential to be a useful in early identification of drug efficacy. The benefits of identifying such markers is well evidenced in the rapid uptake in the use of cardiac MRI in the quantification of left ventricular mass and volumes in drug studies, where the high reproducibility results in a reduction in study size number of 55-93\%, with the observed changes in ventricular measures correlating well with outcomes [17, 18]. CIMT and carotidfemoral pulse wave velocity (cfPWV) have also been used as surrogate markers for assessment of atherosclerosis progression. Of these, CIMT is the best studied, with reported progression rates of $0.0147 \mathrm{~mm} /$ year [19]. However, due to the resolution of ultrasound being in the range of $0.1-0.3 \mathrm{~mm}$, and a 5-10\% measurement error, individual progression monitoring is not possible and the number required for observational studies is still relatively high [20]. Progression rates of cfPWV have previously been reported as $8.1-14.7 \mathrm{~mm} / \mathrm{s} /$ year observed in a population of 483 subjects over a 6 years period, thus also requiring large numbers and long time periods to demonstrate change [21]. The strength of the WB-MRA technique appears to arise in its systemic assessment. When the arterial system was subdivided into five clinically separate areas no significant change over the 3 years was apparent in any of them. It was only when all 31 arterial segments were combined that progression could be observed. This may in part be due to the small number of subjects in our current study meaning significant progression could not be observed in individual arteries or regions. While larger study numbers may allow regional assessment of progression, this detracts from the two strengths of the whole body MRA technique, the first being its systemic assessment of a systemic disease process, and the second being its potential to reduce study sizes by detecting changes in a smaller number of individuals. While whole body CTA has previously been used to derive a similar whole body atheroma score, and has the benefit of providing coronary artery assessment at the same time, it is associated with a high radiation dose and a relatively high contrast dose [22].

The small number of patients who completed the protocol limits the generalisability of the results. However the current study was to determine feasibility rather than to definitively quantify atheroma progression rates. Despite the small number of participants, significant disease progression could still be observed. The validity of our findings is strengthened by the observation that those with atheroma progression were significantly less likely to be prescribed statins and to have higher SAS on their baseline WB-MRA. This is entirely consistent with studies in coronary artery calcium (CAC) scoring - a measure of calcified atheroma burden within the coronaries - showing higher CAC progression in those with higher baseline CAC [23], and lower progression rates in those on statins [24]. While a high drop out rate is problematic and raises concerns about patient acceptance of the technique, a previous study has shown higher acceptance of WB-MRA over digital subtraction angiography and thus the high attrition rate may be due to the high morbidity of the patient population studied, indeed half of our observed attrition rate was due to declining health [25].

Larger studies will be required to verify the reproducibility of these results, and also to explore the applicability in other cardiovascular disease cohorts, especially as PAD is known to have a higher atheroma burden, and therefore is likely to have a higher and therefore more easily measurable progression in atheroma burden. Additionally, the clinical significance of the WB-MRA progression data observed in this study needs to be established.

The current study has several limitations. The population is heterogenous as all clinical referrals for claudication assessment were recruited rather than a specifically defined disease severity. Despite this the majority of the participants were Fontaine IIb, and this heterogeneity also better reflects the spectrum of disease seen in routine clinical practice. In addition our analysis comparing the progression and regression groups could be confounded by the inclusion of participants who underwent interventional procedures, as this group could be considered clinical progressors due to their need for intervention, while those with no intervention and regression would be considered as true (or at least medically induced) regressors. However our results are commensurate with prior work in CAC [23, 24], and the study was neither designed nor of sufficient size to undertake this type of subgroup analysis. Further work is thus required to extricate the true effects of medical intervention. The magnetic field strength used for baseline and 3 years follow-up examinations was different in some cases for operational reasons. All participants were scanned using the $1.5 \mathrm{~T}$ machine at baseline and 6 months, and $70 \%$ of participants were scanned at $3 \mathrm{~T}$ at the final 3 years follow-up. While this could potentially account for some of the temporal changes observed, previous studies have reported no significant differences in stenosis assessment 
between the two field strengths [26]. In addition there were no differences in progression rates between those scanned at $1.5 \mathrm{~T}$ at all visits and those scanned at $3 \mathrm{~T}$ at follow-up.

Whole body magnetic resonance angiography is a lumenographic technique, thus early wall thickening and extra-luminal plaque formation will be missed potentially underestimating disease. Whilst techniques such as isotropic black blood imaging may hold the potential for analysis of early wall thickening and stenosis assessment, this technique has yet to be used and validated in whole body vascular assessment, unlike the current angiographic technique. Furthermore other scores such as the Dukes score and modified Dukes score for invasive coronary angiography and CT coronary angiography have well demonstrated the strength of lumenographic techniques for quantification of disease burden and future prognosis [27].

\section{Conclusion}

Whole body contrast-enhanced magnetic resonance angiography allows monitoring and quantitative assessment of global plaque burden over time. In patients with PAD, significant increases in atheroma burden can be observed over a 3 years period.

Acknowledgments We would like to acknowledge Monica RossMcLean for her assistance in the study.

Funding This study was funded by TMRC Wyeth. JRWM is supported by the Wellcome Trust through the Scottish Translational Medicine and Therapeutics Initiative (Grant No. WT 085664) in the form of a Clinical Research Fellowship.

\section{Compliance with ethical standards}

Conflict of interest $\mathrm{GH}$ has received research grants from Guerbet. JRWM, RDW, PGR, SJG, FK, ADS, JJB declare that they have no conflict of interest.

Ethical approval All procedures performed in studies involving human participants were in accordance with the ethical standards of the institutional and/or national research committee and with the 1964 Helsinki declaration and its later amendments or comparable ethical standards.

Informed consent Informed consent was obtained from all individual participants included in the study.

Open Access This article is distributed under the terms of the Creative Commons Attribution 4.0 International License (http://crea tivecommons.org/licenses/by/4.0/), which permits unrestricted use, distribution, and reproduction in any medium, provided you give appropriate credit to the original author(s) and the source, provide a link to the Creative Commons license, and indicate if changes were made.

\section{References}

1. Bhatt DL, Eagle KA, Ohman EM et al (2010) Comparative determinants of 4-year cardiovascular event rates in stable outpatients at risk of or with atherothrombosis. JAMA 304:1350-1357. doi:10.1001/jama.2010.1322

2. Steg PG, Bhatt DL, Wilson PWF et al (2007) One-year cardiovascular event rates in outpatients with atherothrombosis. JAMA 297:1197-1206. doi:10.1001/jama.297.11.1197

3. Goyen M, Herborn CU, Kröger K et al (2006) Total-body 3D magnetic resonance angiography influences the management of patients with peripheral arterial occlusive disease. Eur Radiol 16:685-691. doi:10.1007/s00330-005-0001-8

4. Roghi A, Palmieri B, Crivellaro W et al (2001) Relationship of unrecognised myocardial infarction, diabetes mellitus and type of surgery to postoperative cardiac outcomes in vascular surgery. Eur J Vasc Endovasc Surg 21:9-16. doi:10.1053/ejvs.2000.1213

5. Hansen T, Wikström J, Johansson LO et al (2007) The prevalence and quantification of atherosclerosis in an elderly population assessed by whole-body magnetic resonance angiography. Arterioscler Thromb Vasc Biol 27:649-654. doi:10.1161/01.ATV. $0000255310.47940 .3 \mathrm{~b}$

6. Gandy SJ, Lambert M, Belch JJF et al (2015) Technical assessment of whole body angiography and cardiac function within a single MRI examination. Clin Radiol. doi:10.1016/j.crad.2015. 02.003

7. Ladd SC, Debatin JF, Stang A et al (2007) Whole-body MR vascular screening detects unsuspected concomitant vascular disease in coronary heart disease patients. Eur Radiol 17:1035-1045. doi:10.1007/s00330-006-0434-8

8. Lehrke S, Egenlauf B, Steen H et al (2009) Prediction of coronary artery disease by a systemic atherosclerosis score index derived from whole-body MR angiography. J Cardiovasc Magn Reson 11:36. doi:10.1186/1532-429X-11-36

9. Bamberg F, Parhofer KG, Lochner E et al (2013) Diabetes mellitus: long-term prognostic value of whole-body MR imaging for the occurrence of cardiac and cerebrovascular events. Radiology 269:730-737. doi:10.1148/radiol.13130371

10. Lundberg C, Johansson L, Barbier CE et al (2013) Total atherosclerotic burden by whole body magnetic resonance angiography predicts major adverse cardiovascular events. Atherosclerosis 228:148-152. doi:10.1016/j.atherosclerosis.2013. 02.015

11. Weir-McCall JR, Khan F, Lambert MA et al (2014) Common carotid intima media thickness and ankle-brachial pressure index correlate with local but not global atheroma burden: a cross sectional study using whole body magnetic resonance angiography. PLoS One 9:e99190. doi:10.1371/journal.pone.0099190

12. Waugh SA, Ramkumar PG, Gandy SJ et al (2009) Optimization of the contrast dose and injection rates in whole-body MR angiography at 3.0T. J Magn Reson Imaging 30:1059-1067. doi:10.1002/jmri.21930

13. Duce SL, Weir-McCall JR, Gandy SJ et al (2015) Cohort comparison study of cardiac disease and atherosclerotic burden in type 2 diabetic adults using whole body cardiovascular magnetic resonance imaging. Cardiovasc Diabetol 14:122. doi:10.1186/ s12933-015-0284-2

14. Goyen M, Herborn CU, Kröger K et al (2003) Detection of atherosclerosis: systemic imaging for systemic disease with whole-body three-dimensional MR angiography-initial experience. Radiology 227:277-282. doi:10.1148/radiol.2271020488

15. Wikström J, Hansen T, Johansson L et al (2008) Ankle brachial index $<0.9$ underestimates the prevalence of peripheral artery occlusive disease assessed with whole-body magnetic resonance 
angiography in the elderly. Acta Radiol 49:143-149. doi:10. 1080/02841850701732957

16. Gassull D, Schulthess D, Suttie S, Houston G (2013) Whole-body magnetic resonance angiography (WBMRA) as a tool for driving efficiency in the cost and treatment of claudication co-morbities. Heal Policy Technol 2:181-187. doi:10.1016/j.hlpt.2013.08.003

17. Kramer DG, Trikalinos TA, Kent DM et al (2010) Quantitative evaluation of drug or device effects on ventricular remodeling as predictors of therapeutic effects on mortality in patients with heart failure and reduced ejection fraction: a meta-analytic approach. J Am Coll Cardiol 56:392-406. doi:10.1016/j.jacc. 2010.05.011

18. Grothues F, Smith GC, Moon JCC et al (2002) Comparison of interstudy reproducibility of cardiovascular magnetic resonance with two-dimensional echocardiography in normal subjects and in patients with heart failure or left ventricular hypertrophy. Am J Cardiol 90:29-34

19. Bots ML, Evans GW, Riley WA, Grobbee DE (2003) Carotid intima-media thickness measurements in intervention studies: design options, progression rates, and sample size considerations: a point of view. Stroke 34:2985-2994. doi:10.1161/01.STR. 0000102044.27905.B5

20. O'Leary DH, Bots ML (2010) Imaging of atherosclerosis: carotid intima-media thickness. Eur Heart J 31:1682-1689. doi:10.1093/ eurheartj/ehq185

21. Benetos A, Adamopoulos C, Bureau JM et al (2002) Determinants of accelerated progression of arterial stiffness in normotensive subjects and in treated hypertensive subjects over a 6-year period. Circulation 105:1202-1207. doi:10.1161/hc1002. 105135

22. Napoli A, Catalano C, Francone M et al (2009) Imaging coronary and extracoronary atherosclerosis: feasibility and impact of whole-body computed tomography angiography. Eur Radiol 19:1704-1714. doi:10.1007/s00330-009-1342-5

23. Yoon H-C, Emerick AM, Hill JA et al (2002) Calcium begets calcium: progression of coronary artery calcification in asymptomatic subjects. Radiology 224:236-241. doi:10.1148/radiol. 2241011191

24. Kronmal RA, McClelland RL, Detrano R et al (2007) Risk factors for the progression of coronary artery calcification in asymptomatic subjects: results from the Multi-Ethnic Study of Atherosclerosis (MESA). Circulation 115:2722-2730. doi:10. 1161/CIRCULATIONAHA.106.674143

25. Nielsen YW, Eiberg JP, Løgager VB et al (2010) Patient acceptance of whole-body magnetic resonance angiography: a prospective questionnaire study. Acta Radiol 51:277-283. doi:10. 3109/02841850903482893

26. Diehm N, Kickuth R, Baumgartner I et al (2007) Magnetic resonance angiography in infrapopliteal arterial disease: prospective comparison of 1.5 and 3 Tesla magnetic resonance imaging. Invest Radiol 42:467-476. doi:10.1097/01.rli.0000262581.52315. ef

27. Min JK, Shaw LJ, Devereux RB et al (2007) Prognostic value of multidetector coronary computed tomographic angiography for prediction of all-cause mortality. J Am Coll Cardiol 50:1161-1170. doi:10.1016/j.jacc.2007.03.067 